\title{
Early Overhearing Avoidance in Wireless Sensor Networks
}

\author{
Siquan $\mathrm{Hu}$ and Mehul Motani \\ Department of Electrical and Computer Engineering, \\ National University of Singapore, 117576 Singapore \\ \{husq,motani\}@nus.edu.sg
}

\begin{abstract}
To reduce the overhearing cost of long preambles in low power listening (LPL), we propose an early overhearing avoidance technique, in which the destination address is embedded into the continuous preamble. The overhearing can be stopped after receiving a short preamble block, which is much earlier than after the whole data packet is received. Based on this idea, a novel low power media access control (MAC) protocol called asynchronous pseudo preamble listening (APPL) is designed to improve the power efficiency of LPL. Both analysis and experiments show that APPL saves more power than LPL, especially on nodes with more neighbors and fewer children.
\end{abstract}

Keywords: Overhearing avoidance, Wireless sensor networks, Medium access control.

\section{Introduction}

Increasing the network lifetime is a key challenge in low power sensor networks. In this scenario, the MAC protocols used must be energy efficient by reducing potential energy wastage. In resource-constrained wireless sensor networks, one of the primary mechanisms to achieve low power operation is duty cycling, in which each sensor node periodically cycles between awake and sleep states.

Most low power MAC protocols proposed for wireless sensor networks can be roughly categorized into scheduling-based and sampling-based approaches, along with hybrid combinations. Scheduling-based protocols include TDMA, FDMA, CDMA and variations such as S-MAC [1], T-MAC [2] and TRAMA [3]. Sampling-based MAC protocols include Aloha with preamble sampling [4, BMAC [5, WiseMAC [6], etc. Hybrid protocols also exist that combine a synchronized protocol with asynchronous sampling [7].

In scheduling-based protocols, a specific schedule of media access is used in time, frequency, or code domains. For instance, in TDMA family of MAC protocols for wireless sensor networks, nodes schedule their radio states in discrete time intervals (slots) and synchronize slots with neighbors allows nodes to only power the radio on when needed, significantly reducing idle listening. Generally, scheduling-based protocols require some kind of synchronization and lack scalability. Scheduling reduces energy cost by ensuring that listeners and transmitters

A. Das et al. (Eds.): NETWORKING 2008, LNCS 4982, pp. 26-35, 2008.

(C) IFIP International Federation for Information Processing 2008 
have a short period to rendezvous and can sleep at other times. Schedule maintenance and idle listening in contention windows cause extra overhead. The sleep interval can be extended to save more power, but this can potentially reduce throughput.

In sampling-based MAC methods, the sensor nodes do not coordinate their wakeup/sleep schedules by explicit synchronization. Rather, nodes periodically wake up and only start receiving data if they detect channel activity by sampling based either on channel energy or successful symbol decoding. Periodic radio sampling allows a node to conserve energy by keeping its radio off most of the time. Although different kinds of schedule synchronization may be introduced into sampling protocols, as a pure asynchronous sampling-based MAC, low power listening (LPL) is still the most attractive protocol in wireless sensor networks. The duty cycles of the sender and receiver can be completely decoupled, which provides great flexibility and scalability. The simplicity of this asynchronous design removes the need for, and the overhead introduced by, synchronized wakeup/sleep schedules. The code size is also reduced which is valuable to the light-weight protocol design requirement for most WSN application, especially body sensor networks.

Unlike scheduling based protocols, which send regular data packets, LPL must send long, expensive messages to ensure that the receiver can receive the payload in the message. In every transmission, the sender sends a preamble before the payload. The preamble length must be at least as long as the receiver's sampling interval. When sharing a common wireless medium, the data transmitted by one node can reach all the other nodes within its transmission range. The receiver will wake up periodically, detect the preamble, and stay awake to receive the data. If the receiver is not the targeted node, the receiving wastes energy, which we refer to as overhearing.

Idle listening is a dominant factor of power inefficiency in many sensor network applications. LPL reduces idle listening successfully by shifting the burden of synchronization to the sender and lowering the radio duty cycle by turning the radio off much of the time. The other source of energy waste is overhearing, which occurs when a node receives packets that are not destined to itself. LPL doesn't address this problem, which can become severe in dense deployments. Reducing the cost of overhearing could improve the power efficiency of LPLbased protocols.

In this paper, we propose an early overhearing avoidance technique to reduce the overhearing cost in LPL. The core idea is that the destination address is embedded into the preamble so that the untargeted nodes can go to sleep quickly after parsing the destination address. Furthermore, we propose an asynchronous pseudo preamble listening (APPL) MAC for wireless sensor networks, which preserves the advantage of asynchronous LPL and reduces the overhead of overhearing based on the pseudo preamble structure. APPL has higher power efficiency than LPL without decreasing the throughput and latency performance.

The remainder of this paper is organized as follows. Section 2 describes related work. The APPL MAC protocol is presented in Section 3 and its performance is 
analytically evaluated in Section 4. Implementation of APPL protocol on Mica2 and the experimental results are described in Section 5. Section 6 concludes this paper.

\section{Related Work}

LPL is an approach where the channel is sampled very briefly for the presence of activity rather than for specific data. The idea of sending a wakeup signal by simply adding preambles in front of each transmitted packet was initially introduced into wireless sensor networks by Hill [8] and El-Hoiydi [4 independently. Hill proposed to periodically sample the energy of analog baseband of the radio and use a low power radio frequency wakeup signal to reduce the duty cycle of the radio. El-Hoiydi's Aloha with preamble sampling protocol proposed that packets be sent with long preambles to match the channel check period. B-MAC [5] implemented LPL in TinyOS with a well-defined interface for applications to perform fine-grained control over the MAC.

Several groups have improved specific aspects of LPL. WiseMAC [6] is an iteration on Aloha with preamble sampling and it improves previous LPL protocols by reducing the preamble length after an initial unicast packet with a long preamble. The receiver piggybacks its next sampling time in the ACK packet, allowing the sender to send the next packet with a short preamble. SP [9] similarly proposes a scheme for a node to piggyback its channel sampling schedule when it sends a data packet with the long LPL preamble. Both WiseMAC and SP have limitations caused by clock drift. UBMAC [10] further reduces the long preambles in B-MAC by precisely estimating the clock drift between packet transmissions. It requires fine-grain time synchronization which requires high-resolution timers and has very high CPU cost.

Halkes et al. 7 study the benefit of directly utilizing LPL in the listening intervals of S-MAC and T-MAC. SCP-MAC [11] trades off the penalty of long LPL preambles with the cost of synchronizing schedules based on worst-case clock drift. It also adjusts the sampling interval based on traffic.

Rather than introducing schedule either by fine or coarse schedule, our work differs from each of the above schemes to improve LPL's power efficiency by overhearing avoidance without losing the flexibility of long preambles. SCP-MAC does include a kind of overhearing avoidance for LPL which is based on MAC headers. To do this, a receiver examines the destination address of a packet immediately after receiving its $\mathrm{MAC}$ header, before completely receiving the packet. If it is a unicast packet destined to another node, it immediately stops the reception and places the radio to sleep. Since the decision can only be made after the header is received and the LPL preamble is much longer than the real packet, the power saved is very limited.

Our work is different from X-MAC [12, although both of them embed the destination address into preamble. X-MAC use a series of strobe preambles which are separated by slots trying to receive an ACK from target nodes. At the sender side, the cost is increased by frequent radio switching between transmitting and 
receiving states. It also has difficulty in supporting broadcast or multicast because of lack of coordination among the sender and multiple receivers. Our APPL approach uses the continuous preamble of LPL and restructures it into continuous sequence of Pseudo Preambles, which preserve the asynchronous advantage of LPL as much as possible while reducing the cost of overhearing.

Miller and Vaidya [13] also propose to include the destination address in the wakeup packet through the primary channel of a two-radio architecture. However, our work doesn't require such special hardware and APPL can work on current practical WSN platforms, most of which are using a single radio chip.

\section{Design of Asynchronous Pseudo Preamble Listening}

The design goal of APPL is to preserve the advantage of asynchronous duty cycling techniques and to improve LPL's power efficiency by early overhearing avoidance. Low-power listening is an approach to reduce idle listening, by introducing a duty-cycle, which operates at the physical layer. The basic idea of low-power listening is to shift the cost from the receiver to the transmitter by increasing the length of the preamble. This allows the receiver to periodically turn on the radio to sample for incoming data, and detect if a preamble is present or not. If it detects a preamble, it will continue listen until the start symbol arrives and the message can be properly received. If no preamble is detected the radio is turned off again until the next sample.

LPL's inherent asynchronous nature makes it preferable to synchronized protocols in terms of energy efficiency, latency, and throughput. In part, this is because they do not incur overhead due to synchronization. In addition, asynchronous techniques do not have to share schedule information and only stay awake long enough to sample the radio when they are not receiving or transmitting data. Hence, the awake period can be significantly shorter than that of synchronized methods. With a shorter awake period, asynchronous protocols can wake up more often while still maintaining a low duty cycle. Consequently, they experience reduced latency and higher throughput.

In the networking stack, power efficiency is not only involved at any single layer. To achieve the primary goal of energy saving, cross-layer design is becoming more and more important. Most previous works make use of parameters propagated from upper layers to adapt the medium access protocol. Our work applies cross-layer design in a different way. APPL MAC exploits the physical layer's potential of power saving.

In APPL, to save the cost of overhearing for untargeted nodes, we replace the long preamble of LPL with a serial of short pseudo preamble blocks followed by one short true preamble. The last true preamble is followed by the start symbol and MAC header. All pseudo preamble blocks, true preamble, start symbol are a continuous bit stream.

Each pseudo preamble block is composed of three parts. The first part is the ordinary preamble bit pattern; the length of the preamble bit pattern should be the minimum required length of preamble in $100 \%$ duty cycle LPL, i.e. no 


\begin{tabular}{|l|l|l|l|l|l|}
\hline Oxaa & 0xaa & $\cdots$ & 0xaa & PSS & Dest \\
\hline
\end{tabular}

Fig. 1. Structure of the pseudo preamble block for Mica2. (PSS is the pseudo start symbol, Dest is the destination address of current packet.)

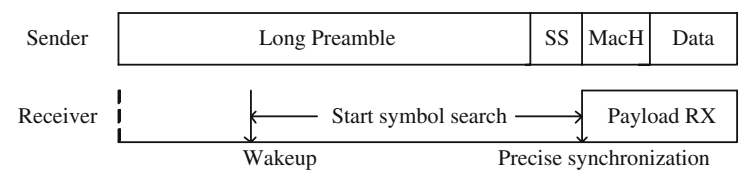

(a) Timeline of LPL using long preamble

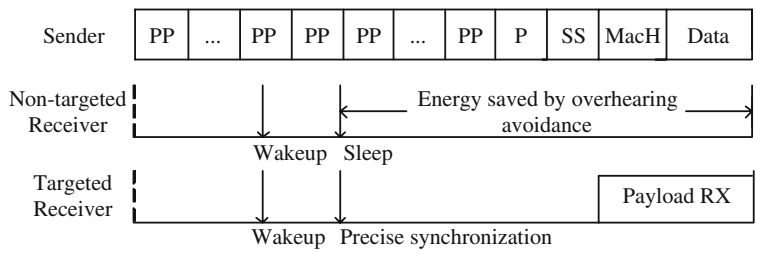

(b) Timeline of APPL using pseudo preamble

Fig. 2. Comparison of the timelines of LPL and APPL. (SS is the start symbol, PP is the pseudo preamble block, $\mathrm{P}$ is the short standard preamble, $\mathrm{MacH}$ is the MAC header.)

power management. The second part is a pseudo start symbol which is different from the regular start symbol. The pseudo start symbol is used to determine the bit shift of followed address information. The third part is the destination address information. Fig. 1 is an example of pseudo preamble block which using '010101...' bit pattern such as CC1000 radio chip.

Similar to LPL, each node in APPL periodically wakeups and samples the preamble. If the radio strength is strong enough, the radio transfer to RX state and receives the preamble. From the short pseudo preamble block or the true preamble followed by the MAC header, the destination address of the packet can be identified. If the local node doesn't match the destination address, it aborts the reception in progress and goes to sleep quickly. If local node is the target node, then it will continue to detect the start symbol to receive the real data. Similar to the standard start symbol, the pseudo start symbol can be used to determine the bit shift of the bytes in the preamble block, so the cost of bit synchronization doesn't increase in APPL. Fig. 2]illustrates the timelines of LPL and APPL.

\section{Performance Analysis}

In this section we analyze the energy performance of LPL and APPL. Both LPL and APPL are asynchronous, giving them flexibility and simplicity without synchronization overhead. We will only analyze their energy efficiency. Our analysis 
Table 1. Parameters of Mica2 platform

\begin{tabular}{|c|c|c|}
\hline Symbol & Value & Description \\
\hline$P_{t x}$ & $60 \mathrm{~mW}$ & Power in transmitting \\
\hline$P_{r x}$ & $45 \mathrm{~mW}$ & Power in receiving \\
\hline$P_{\text {sampling }}$ & $15.5 \mathrm{~mW}$ & Power in radio sampling \\
\hline$P_{\text {sleep }}$ & $0.09 \mathrm{~mW}$ & Power in sleeping \\
\hline$T_{B}$ & $0.000416 \mathrm{~s}$ & Time to TX/RX a byte \\
\hline$T_{\text {sampling }}$ & $0.00255 \mathrm{~s}$ & Time to sample radio \\
\hline$T_{\text {ch }}$ & $0.125 \mathrm{~s}$ & Radio Check Interval \\
\hline$T_{\text {gen }}$ & $180 \mathrm{~s}$ & Data generate Interval \\
\hline$L_{\text {packet }}$ & 36 bytes & Packet Length \\
\hline$L_{\text {preamble }}$ & 308 bytes & LPL preamble length \\
\hline$L_{p p}$ & 8 bytes & pseudo preamble block length \\
\hline$N_{p p}$ & 38 & number of pseudo preamble blocks \\
\hline$n$ & 10 & neighborhood density \\
\hline
\end{tabular}

is based on a multi-hop network model where each node periodically generates packets at a fixed interval. Each node in the network will sample the channel at a uniform rate and detect the preamble when each of its $n$ neighbors transmits a packet. In the configuration using LPL, the node will receive the data regardless of the packet's destination. Whereas in configuration using APPL protocol, the node will determine whether to receive the data or go to sleep based on the detected preamble. The model parameters are given in Table 1 and the typical values on Mica2 platform are also listed (the values are sourced from [5] and the voltage is assumed to be 3 Volts).

Consider a node $i$ in the network, the number of neighbors surrounding the node $i$ is denoted by $n$, which is neighborhood density of node $i$. Since our interest is power efficiency at the MAC layer, we will ignore the details of the routing layer. Furthermore, without loss of generality, we use a function $c(i)$ to represent the number of children of node $i$ in the multihop network. To simplify the analysis, we will ignore the boundary condition in which one node among neighbors of node $i$ will forward the message out of the neighborhood of $i$.

Our analysis focuses on the energy consumption by the motes, including the radio and $\mathrm{CPU}$; we do not model the sensors. There are four stable radio states: transmitting, receiving, sampling and sleeping. The power (energy per unit time) drawn in each state is denoted by $P_{t x}, P_{r x}, P_{\text {sampling }}$ and $P_{\text {sleep }}$, respectively. The energy consumption of the mote is determined by how much time it spends in transmitting, receiving, sampling and sleeping, denoted as $t_{t x}, t_{r x}, t_{\text {sampling }}$ and $t_{\text {sleep }}$ respectively for LPL, and denoted as $t_{t x}^{\prime}, t_{r x}^{\prime}, t_{\text {sampling }}^{\prime}$ and $t_{\text {sleep }}^{\prime}$ respectively for APPL. In our analysis, all these time values are normalized to one second. They represent the fractions of time in one second the node is in the 


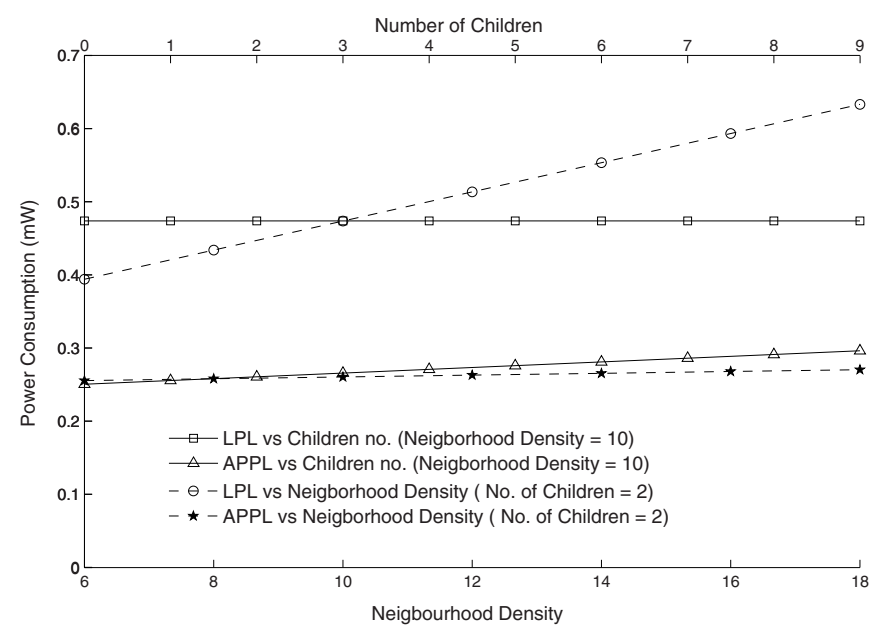

Fig. 3. Power consumption estimation

different states. For both LPL and APPL, the expected energy consumption per node, is the sum of the expected energy spent in each state:

$$
E=E_{t x}+E_{r x}+E_{\text {sampling }}+E_{\text {sleep }} .
$$

For LPL,

$$
E_{l p l}=P_{t x} t_{t x}+P_{r x} t_{r x}+P_{\text {sampling }} t_{\text {sampling }}+P_{\text {sleep }} t_{\text {sleep }} .
$$

For APPL,

$$
E_{\text {appl }}=P_{t x} t_{t x}^{\prime}+P_{r x} t_{r x}^{\prime}+P_{\text {sampling }} t_{\text {sampling }}^{\prime}+P_{\text {sleep }} t_{\text {sleep }}^{\prime} .
$$

So we only need to know the time spent on each state to deduce the total energy. Actually the time spent on channel sampling is the same for LPL and APPL when using the same channel check interval. So

$$
t_{\text {sampling }}=t_{\text {sampling }}^{\prime}=T_{\text {sampling }} / T_{\text {ch }} .
$$

To compare the energy efficiency of LPL and APPL, we use the same length of preamble for both. So

$$
L_{\text {preamble }}=\left(N_{p p}+1\right) L_{p p}-4,
$$

which assumes the length of pseudo start symbol and true start symbol is 2 bytes and the address is 2 bytes. So the time spent on transmitting in LPL and APPL equal to

$$
t_{t x}=t_{t x}^{\prime}=\left(L_{\text {preamble }}+2+L_{\text {packet }}\right) T_{B} / T_{\text {gen }} .
$$

The energy spent on receiving is varying because start point of receiving is random; however, its expected value can be estimated. Let $L_{r x}$ denotes the 
expected value of received bytes, then $L_{r x}=0.5 L_{\text {preamble }}+2+L_{\text {packet }}$. For LPL,

$$
t_{r x}=n L_{r x} T_{B} / T_{g e n}
$$

For APPL,

$$
t_{r x}^{\prime}=\left(1.5(n-c(i)) L_{p p}+c(i) L_{r x}\right) T_{B} / T_{g e n} .
$$

And the remaining time in 1 second is the sleep time. So for LPL, the sleep time is

$$
t_{\text {sleep }}=1-t_{t x}-t_{r x}-t_{\text {sampling }} .
$$

And for APPL, the sleep time is

$$
t_{\text {sleep }}^{\prime}=1-t_{t x}^{\prime}-t_{r x}^{\prime}-t_{\text {sampling }}^{\prime} .
$$

Then the energy spent in 1 second for LPL can be computed from formula $(1)(3)(4)(5)(6)(8)$, and from formula $(2)(3)(4)(5)(7)(9)$ to get APPL's total energy in 1 second.

From (6) and (7), we can know if the children number of node i decreases, APPL's time spent on receiving will become smaller so more energy is saved. However, LPL's receiving time doesn't change as the number of children changes. In Fig. 3, the energy spent on 1 second for different $c(i)$ are shown as real lines. The dotted lines in Fig. 3 show the effect of neighborhood density; we can see that the higher density, more energy saved by APPL compared to LPL.

To match the pulse check interval, APPL's total preamble length including all pseudo preamble blocks are the same as LPL's preamble provided that they have the same pulse check interval. Both of them have the same latency and throughput performance. At most one packet can flow through a node in a pulse check interval. This means APPL improves the energy performance of LPL MAC without decreasing the latency and throughput.

\section{$5 \quad$ Experimental Evaluation}

The main contribution of our work is to propose to embed the destination address into the preamble so that the overhearing can be stopped after the destination address is parsed, which is much earlier than after the whole data packet is received. We call this early overhearing avoidance, and have proposed a novel low power MAC, APPL, to improve the power efficiency of LPL. The analytical performance computations show that APPL saves more power than LPL, especially when neighborhood density is high, and the saving is more significant in those nodes which have fewer children.

We have implemented both LPL and APPL protocol on the Mica2 platform on TinyOS to validate our analysis. The parameters are the same as those listed in Table 1 except for neighborhood size. We use the deployment topology shown

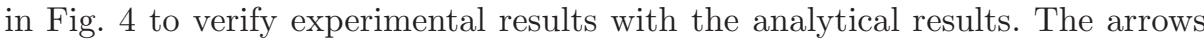
in the topology map represent the routing from a child node to its parent node. Node 0 is the base station. Nodes $1-6$ are deployed in the radio range of each 


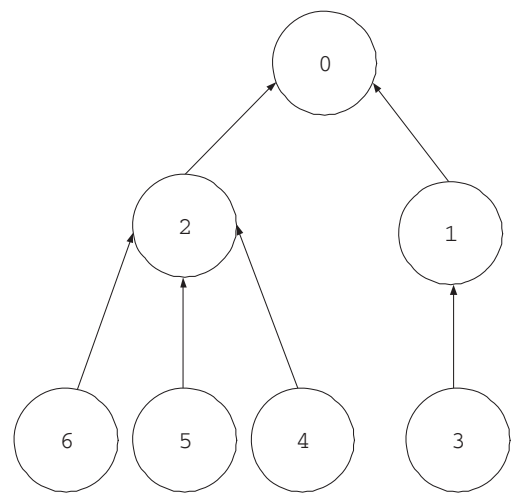

Fig. 4. The topology map of the test

Table 2. The power consumption results

\begin{tabular}{|c|c|c|c|}
\hline Power Consumption $(\mathrm{mW})$ & Node 2 & Node 1 & Node3-6 \\
\hline LPL & 0.54 & 0.48 & 0.46 \\
\hline APPL & 0.48 & 0.35 & 0.28 \\
\hline
\end{tabular}

other to test the effect of overhearing. Only node 1 and node 2 are in the radio range of base station. The topology used is static, which is sufficient for MAC layer performance evaluation. The base station is on full power state and doesn't generate any packets. The neighborhood size of nodes 1-6 is 6 . The number of children of each node is shown in the map. As noted before, $c(i)$ is the number of children of node $\mathrm{i}$, so $c(1)=1, c(2)=3$, for other node $i(i=3,4,5,6), c(i)=0$, which means they are leaf nodes.

We measure the energy consumption at each node by recording the time spent by the radio in the different states and normalize them to 1 second. The experiment results are averaged over a 1 hour running time and listed in Table 2 For leaf nodes 3-6, the energy efficiency has been increased by $39 \%$; for node 1 who has 1 child, the increased efficiency is $27.1 \%$, and node 2 with 3 children is $11 \%$. The experiment matches well with our analysis in section IV that APPL outperforms LPL especially for nodes who have less children.

\section{Conclusion}

This paper has presented an early overhearing avoidance technique by embedding the destination address into the preamble. We also evaluated a novel MAC, APPL, which uses the early overhearing avoidance technique to improve the power efficiency of LPL. Both analytical computations and experimental evaluation show that APPL is more power efficient than LPL especially on nodes with fewer children and more neighbors. Though APPL builds upon LPL, we 
believe that adding a small amount of information into the long sparse preamble and exploiting early overhearing avoidance will improve the performance of any underlying preamble sampling-based MAC protocol.

\section{Acknowledgment}

This work was supported by the EHS-II project funded by A*STAR, Singapore.

\section{References}

1. Ye, W., Heidemann, J.S., Estrin, D.: An energy-efficient MAC protocol for wireless sensor networks. In: INFOCOM, pp. 1567-1576 (2002)

2. van Dam, T., Langendoen, K.: An adaptive energy-efficient MAC protocol for wireless sensor networks. In: ACM SenSys. pp. 171-180 (2003)

3. Rajendran, V., Obraczka, K., Garcia-Luna-Aceves, J.J.: Energy-efficient collisionfree medium access control for wireless sensor networks. In: ACM SenSys. pp. 181-192 (2003)

4. El-Hoiydi, A.: Aloha with preamble sampling for sporadic traffic in ad hoc wireless sensor networks. In: IEEE ICC., pp. 3418-3423 (2002)

5. Polastre, J., Hill, J., Culler, D.: Versatile low power media access for wireless sensor networks. In: ACM SenSys. pp. 95-107 (2004)

6. El-Hoiydi, A., Decotignie, J.D.: WiseMAC: An ultra low power MAC protocol for multi-hop wireless sensor networks. In: First Int. Workshop on Algorithmic Aspects of Wireless Sensor Networks, July 2004, pp. 18-31 (2004)

7. Halkes, G.P., van Dam, T., Langendoen, K.G.: Comparing energy-saving MAC protocols for wireless sensor networks. Mob. Netw. Appl. 10(5), 783-791 (2005)

8. Hill, J., Culler, D.: Mica: A wireless platform for deeply embedded networks. IEEE Micro. 22(6), 12-24 (2002)

9. Polastre, J., Hui, J., Levis, P., Zhao, J., Culler, D., Shenker, S., Stoica, I.: A unifying link abstraction for wireless sensor networks. In: ACM SenSys. pp. 76-89 (2005)

10. Ganeriwal, S., Ganesan, D., Shim, H., Tsiatsis, V., Srivastava, M.B.: Estimating clock uncertainty for efficient duty-cycling in sensor networks. In: ACM SenSys. pp. 130-141 (2005)

11. Ye, W., Silva, F., Heidemann, J.: Ultra-low duty cycle MAC with scheduled channel polling. In: ACM SenSys. pp. 321-334 (2006)

12. Buettner, M., Yee, G.V., Anderson, E., Han, R.: X-MAC: a short preamble MAC protocol for duty-cycled wireless sensor networks. In: ACM SenSys. pp. 307-320 (2006)

13. Miller, M.J., Vaidya, N.H.: A MAC protocol to reduce sensor network energy consumption using a wakeup radio. IEEE Trans. Mobile Comput. 4(3), 228-242 (2005) 